\title{
Adjuvant chemotherapy and follow-up for recurrences in localized testicular cancer
}
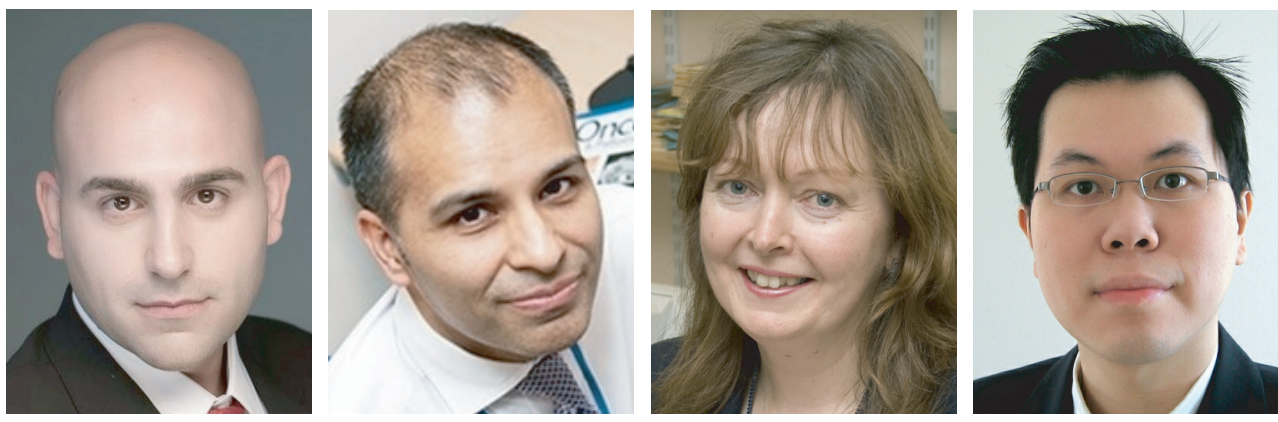

Suliman Boulos ${ }^{*, 1}$, Danish Mazhar' ${ }^{1}$ Anne Y Warren² \& Han Hsi Wong1

First draft submitted: 27 January 2017; Accepted for publication: 23 February 2017; Published online: 8 May 2017

Patients diagnosed with testicular germ cell tumor (seminoma and nonseminomatous germ cell tumor [NSGCT]) have one of the best prognoses of malignant neoplasms [1]. Following orchiectomy for stage I (testis confined) disease, management options include adjuvant chemotherapy and active surveillance. Although chemotherapy is associated with lower risk of relapse compared with surveillance, the long-term survival is excellent in both cases given the tumor's exquisite sensitivity to cytotoxic drugs, particularly to bleomycin, etoposide and cisplatin (BEP).

\section{Adjuvant chemotherapy: seminoma}

Tumor size of $>4 \mathrm{~cm}$ and rete testis invasion were previously shown to be associated with increased risk of relapse for stage I seminoma, with rates of 31.5, 15.9 and $12.2 \%$ in those with both-, one- and no-risk factors, respectively [2]. These are now often utilized to facilitate decision making between adjuvant chemotherapy and active surveillance. Single-dose carboplatin (AUC7) has become a standard treatment for stage I seminoma following the MRC TE19/EORTC 30982 trial which demonstrated noninferiority of carboplatin compared with adjuvant radiotherapy [3]. This study did not divide patients according to their risk groups, although most centers would now recommend active surveillance for low-risk patients and only consider carboplatin for those with risk factors [4-6]. A number of other studies have reported overall recurrence rates of $1.5-6.2 \%$ following adjuvant carboplatin compared with $8.2-14.3 \%$ with active surveillance [7-11]. Differences in the efficacy of carboplatin could be related to the patient number, regimens (e.g., one vs two cycles) and underdosing of carboplatin.

A recent series suggested that patients who received carboplatin relapsed later than those on active surveillance (median of 19 vs 14 months; relapse risk after 3 years was 15 vs $8 \%$ ) [4,12]. In addition, $15 \%$ of patients treated for their first relapse after adjuvant carboplatin experienced a second relapse,

\section{KEYWORDS}

- follow-up • recurrence • testicular cancer

'Department of Oncology, Addenbrooke's Hospital, Cambridge University Hospitals NHS Foundation Trust, Cambridge Biomedical Campus, Hills Road, Cambridge, CB2 0QQ, UK

2Department of Pathology, Addenbrooke's Hospital, Cambridge University Hospitals NHS Foundation Trust, Cambridge Biomedical Campus, Hills Road, Cambridge, CB2 0QQ, UK

*Author for correspondence: Tel.: +972 50 6770650; Fax: +44 1223 217020; medboulos@gmail.com 
compared with only $4 \%$ of patients on active surveillance. This could reflect more aggressive disease in patients who received carboplatin, as this is primarily used for those with risk factors. A number of other studies have also suggested that the risk of recurrence remains high even after adjuvant carboplatin (up to $12.5 \%$ ) for high-risk patients $[6,8,9,11,13]$. Clinicians and patients should be aware of these and a more stringent and personalized follow-up plan might have to be considered.

\section{Adjuvant chemotherapy: NSGCT}

BEP is the adjuvant treatment of choice for stage I NSGCT. Given the increased risk of toxicities with this regimen, it is normally offered to high-risk patients (tumors showing lymphovascular invasion), as the relapse risk could be as high as $50 \%$. Initial studies have recommended two cycles of BEP, but one cycle is increasingly being used to improve its risk-benefit ratio [14-16] . Relapse rate after one cycle of BEP in patients with lymphovascular invasion has been reported to be $3.2 \%$ compared with $41.7 \%$ for surveillance [16].

\section{Follow-up for stage I disease: is there an optimal approach?}

Given the rarity, curability and mostly predictable nature of testicular cancer, its management pathway is highly protocolized. After initial treatment, patients are followed up by hospital practitioners according to locally agreed guidelines, which are largely based on expert opinion and consensus. The schedule and methods of assessment vary between institutions, although they would normally include history/examination, serum tumor markers, chest $\mathrm{x}$-ray and computed tomography (CT). The value of some of these assessment methods was highlighted in a study by Kollmannsberger et al. which involved 2483 stage I testicular cancer patients managed by active surveillance [4]. CT was found to be most effective in detecting first relapses (63\% overall; $87 \%$ for seminoma; and $44 \%$ for NSGCT), followed by tumor markers (28\% overall; $3 \%$ for seminoma; $48 \%$ for NSGCT). Overall, chest $x$-ray and physical examination detected only $0.5 \%$ of recurrences each.

Given that the vast majority of relapses are detected by imaging and blood tests, do patients really need to see a hospital specialist for followup, especially when physical examination is unlikely to be productive? Is it time to redesign the way we follow-up patients?
The incidence of cancer is rising globally, including testicular cancer [1]. More patients are living with and beyond the diagnosis of cancer, and hospitals and clinicians are being overwhelmed by the number of patients on long-term follow-up. From the patients' perspectives, they have to take time off work, travel long distances and put up with the long wait for clinic appointments. The financial and emotional costs could be substantial. As such, patients and the health service could benefit from alternative models of follow-up to provide a safe and effective approach while reducing the burden on both parties. For example, while routine scans could be organized by the hospital team, patients could be followed up by telephone interviews conducted by a trained nurse, when symptoms could be reported and test results are conveyed and discussed. Alternatively, a shared care arrangement could be organized with the patient's general practitioner, who could also provide invaluable input on the management of post-treatment late effects and psychosocial issues. The increasing use of emails and smartphone apps has also opened a range of possibilities in which remote follow-up could be conducted. The UK's National Cancer Research Institute Testis Cancer Clinical Studies Group is currently planning a randomized trial to explore the use of remote follow-up for testicular cancer patients.

No single follow-up plan is appropriate for all patients, and remote follow-up is not without its issues. With remote follow-up, where does the responsibility lie - the patient, general practitioner, nurse or oncologist? Some argue that keeping patient clinic appointments is a better way of ensuring patients are not lost to follow-up; this is a particular concern with young patients who might be less compliant [17]. If an investigation suggests disease recurrence, the results should ideally be discussed with the patient face-to-face rather than by other means of communication. Although physical examination is unlikely to be helpful in detecting relapses, it still has a role in rare cases of second primary tumor in the remaining testis or in unusual sites of recurrence. What about testicular self-examination? Although often recommended by clinicians, there is no evidence that this is of benefit [18]. One study has suggested that even in men with a strong family history, and therefore at higher risk of developing testicular cancer, the practice of regular self-examination is less than 50\% [19]. Problems with self-examination also include the lack of early palpable lesion, no formal education on examination technique, 
and its low sensitivity and specificity even if this is performed by a clinician.

\section{A rare case of recurrences after adjuvant chemotherapy}

This is an actual patient of ours and the case highlights the fact that standard follow-up protocols do not fit everyone. It describes a presentation of multiple recurrences of seminoma in the contralateral paratesticular region. It is unusual for a number of reasons, including the sites of relapse, that recurrence was multiple and rapid despite adjuvant chemotherapy, and that CT and tumor markers during routine follow-ups were unhelpful. It was patient self-examination that prompted further investigations and subsequent diagnosis.

A 45-year-old man underwent left orchiectomy for a testis-confined tumor in September 2013. His tumor markers were negative. Histology showed a $55-\mathrm{mm}$ seminoma with rete testis involvement. He received one cycle of adjuvant carboplatin (AUC7) in November 2013 and was then followed up in clinic. Written informed consent was obtained from the patient for the publication of this case report.

In June 2015, he identified a lump near his right testis for which ultrasound demonstrated a normal testis and two paratesticular lesions measuring 2.5 and $1.5 \mathrm{~cm}$, respectively. These were solitary lesions with no evidence of distant metastasis on CT. The lesions were excised and histopathological analysis confirmed metastatic seminoma. His tumor markers remained normal. He was given two cycles of BEP which completed in September 2015.

In March 2016, less than a year after his chemotherapy, he felt two lumps within his scrotum. Ultrasound identified two nodules near the right epididymal tail, while the testis remained normal. This was the only site of disease and tumor markers were normal. The lesions were removed and again appeared to be seminoma. A month later another lesion was found at the base of his penis - excision showed seminoma with involved margins. A repeat staging CT was normal.

Three weeks after his third surgery, he felt another lump on the right base of his penis. Orchiectomy of the remaining testis was never performed in all these recurrences, given its persistently normal appearance on ultrasound. However, given the multiple regional recurrences, he was treated with radiotherapy to include the pelvic nodes and testicular region (45 Gy in 25 fractions). Four weeks after radiotherapy, the lump at the base of his penis was no longer palpable and follow-up ultrasound was normal at 4, 8 and 12 weeks postradiotherapy.

\section{Conclusion}

Stage I testicular cancer carries an excellent prognosis after orchiectomy, and adjuvant chemotherapy can be considered to reduce the risk of relapse. Given the high curability of the disease, detection of future recurrences is vital. In this aspect, follow-up protocols are helpful but could be further optimized to improve patient experience and reduce the demand of hospital services, including the judicious use of investigations and consideration of remote follow-ups. There is, however, no one-size-fits-all approach, as demonstrated by our case of rare multiple paratesticular recurrences despite adjuvant chemotherapy.

\section{Financial \& competing interests disclosure}

The authors have no relevant affiliations or financial involvement with any organization or entity with a financial interest in or financial conflict with the subject matter or materials discussed in the manuscript. This includes employment, consultancies, honoraria, stock ownership or options, expert testimony, grants or patents received or pending, or royalties.

No writing assistance was utilized in the production of this manuscript.

\section{References}

Papers of special note have been highlighted as:

- of interest; $\bullet \bullet$ of considerable interest

1 Hanna NH, Einhorn LH. Testicular cancer - discoveries and updates. N. Engl. J. Med. 371(21), 2005-2016 (2014).

- A comprehensive review of testicular cancer and its management.

2 Warde P, Specht L, Horwich A et al. Prognostic factors for relapse in stage I seminoma managed by surveillance: a pooled analysis. J. Clin. Oncol. 20(22), 4448-4452 (2002).

- An important analysis of prognostic factors in stage I seminoma.

3 Oliver RTD, Mead GM, Rustin GJS et al. Randomized trial of carboplatin versus radiotherapy for stage I seminoma: mature results on relapse and contralateral testis cancer rates in MRC TE19/EORTC 30982 study (ISRCTN27163214). J. Clin. Oncol. 29(8), 957-962 (2011).
- A landmark study on the use of adjuvant carboplatin for stage I seminoma.

4 Kollmannsberger C, Tandstad T, Bedard PL et al. Patterns of relapse in patients with clinical stage I testicular cancer managed with active surveillance. J. Clin. Oncol. 33(1), 51-57 (2015).

- A large study of stage I testicular cancer managed by active surveillance, which provides interesting information including the risks and patterns of relapse and the value of routine investigations. 
5 Aparicio J, Maroto P, García del Muro X et al. Prognostic factors for relapse in stage I seminoma: a new nomogram derived from three consecutive, risk-adapted studies from the Spanish Germ Cell Cancer Group (SGCCG). Ann. Oncol. 25(11), 2173-2178 (2014).

6 Tandstad T, Ståhl O, Dahl O et al. Treatment of stage I seminoma, with one course of adjuvant carboplatin or surveillance, risk-adapted recommendations implementing patient autonomy: a report from the Swedish and Norwegian Testicular Cancer Group (SWENOTECA). Ann. Oncol. 27(7), 1299-1304 (2016).

7 Tandstad T, Smaaland R, Solberg A et al. Management of seminomatous testicular cancer: a binational prospective populationbased study from the Swedish norwegian testicular cancer study group. J. Clin. Oncol. 29(6), 719-725 (2011).

8 Chau C, Cathomas R, Wheater M et al. Treatment outcome and patterns of relapse following adjuvant carboplatin for stage I testicular seminomatous germ-cell tumor: results from a 17-year UK experience. Ann. Oncol. 26(9), 1865-1870 (2015).

9 Dieckmann K-P, Dralle-Filiz I, Matthies C et al. Testicular seminoma clinical stage 1: treatment outcome on a routine care level. J. Cancer Res. Clin. Oncol. 142(7), 1599-1607 (2016).

10 Diminutto A, Basso U, Maruzzo M et al. Adjuvant carboplatin treatment in
115 patients with stage I seminoma: retrospective multicenter survey. Clin. Genitourin. Cancer 14(2), e161-e169 (2016).

11 Cohn-Cedermark G, Stahl O, Tandstad T, SWENOTECA. Surveillance vs. adjuvant therapy of clinical stage I testicular tumors - a review and the SWENOTECA experience. Andrology 3(1), 102-110 (2015).

- An excellent review on the management of stage I testicular cancer.

12 Fischer S, Tandstad T, Wheater M et al. Outcome of men with relapse after adjuvant carboplatin for clinical stage I seminoma. J. Clin. Oncol. 35(2), 194-200 (2017).

-• A recent publication which provides useful insight into the patterns of relapse after previous adjuvant carboplatin for stage I testicular seminoma.

13 Ondrusova M, Ondrus D, Miskovska V et al. Management of clinical stage I testicular seminoma: active surveillance versus adjuvant chemotherapy. Int. Urol. Nephrol. 47(7), 1143-1147 (2015).

14 Albers P, Siener R, Krege S et al. Randomized Phase III trial comparing retroperitoneal lymph node dissection with one course of bleomycin and etoposide plus cisplatin chemotherapy in the adjuvant treatment of clinical stage I nonseminomatous testicular germ cell tumors: AUO trial AH 01/94. J. Clin. Oncol. 26(18), 2966-2972 (2008).
- A large randomized trial demonstrating the benefit of adjuvant bleomycin, etoposide and cisplatin chemotherapy in stage I nonseminomatous germ cell tumor.

15 Vidal AD, Thalmann GN, KaramitopoulouDiamantis E, Fey MF, Studer UE. Long-term outcome of patients with clinical stage I high-risk nonseminomatous germ-cell tumors 15 years after one adjuvant cycle of bleomycin, etoposide, and cisplatin chemotherapy. Ann. Oncol. 26(2), 374-377 (2015).

16 Tandstad T, Ståhl O, Håkansson U et al. One course of adjuvant BEP in clinical stage I nonseminoma mature and expanded results from the SWENOTECA group. Ann. Oncol. 25(11), 2167-2172 (2014).

17 Endo T, Kawai K, Kamba T et al. Risk factors for loss to follow-up during active surveillance of patients with stage I seminoma. Jpn J. Clin. Oncol. 44(4), 355-359 (2014).

18 U.S. Preventive Services Task Force. Screening for testicular cancer: US. Preventive Services Task Force reaffirmation recommendation statement. Ann. Intern. Med. 154(7), 483-486 (2011).

19 Vadaparampil ST, Moser RP, Loud J, Peters JA, Greene MH, Korde L. Factors associated with testicular self-examination among unaffected men from multiple-case testicular cancer families. Hered. Cancer Clin. Pr. 7(1), 11 (2009). 